\title{
Demonstration of an FSO/RF hybrid- communication system on aeronautical and space applications
}

Rödiger, Benjamin, Ginthör, David, Pacheco Labrador, Jorge, Ramirez, Julio, Schmidt, Christopher, et al.

Benjamin Rödiger, David Ginthör, Jorge Pacheco Labrador, Julio Ramirez, Christopher Schmidt, Christian Fuchs, "Demonstration of an FSO/RF hybridcommunication system on aeronautical and space applications," Proc. SPIE 11506, Laser Communication and Propagation through the Atmosphere and Oceans IX, 1150603 (22 August 2020); doi: 10.1117/12.2567034

SPIE. Event: SPIE Optical Engineering + Applications, 2020, Online Only 


\title{
Demonstration of an FSO/RF Hybrid-Communication System on Aeronautical and Space Applications
}

\author{
Benjamin Rödiger*a , David Ginthör ${ }^{\mathrm{a}}$, Jorge Pacheco Labrador ${ }^{\mathrm{a}}$, Julio Ramirez ${ }^{\mathrm{a}}$, Christopher \\ Schmidt ${ }^{\mathrm{a}}$, Christian Fuchs ${ }^{\mathrm{a}}$ \\ ${ }^{a}$ German Aerospace Center (DLR), Institute of Communications and Navigation, Muenchener Str. \\ 20, 82234 Wessling, Germany
}

\begin{abstract}
The generated amount of data on high flying platforms like aircrafts, satellites and Unmanned Aerial Vehicles (UAV) increases continuously, due to the technical improvement of modern sensor systems. The resulting demands for higher data rates on airborne and space platforms motivates the development of Laser Communication terminals for aircrafts and satellites in the last years. DLR's Institute of Communications and Navigation has a successful track record in developing Free Space Optical (FSO) terminals for flying platforms like stratospheric balloons, aircrafts and small satellites to transfer data from moving platforms down to earth in real-time.

Beside the advantages of FSO such as high data rates and a secure transfer channel against Radio Frequency (RF) interferences, a direct line of sight is mandatory for a successful link. Traditional RF-Communication is more robust and less effected by atmospheric disturbances or weather conditions. Thus, new system concepts have been developed to benefit from the provided high data rates of the FSO and the reliability of RF-Communication technologies. As part of this trend, DLR has developed and demonstrated a Hybrid FSO/RF-communication system capable of overcoming the spurious effects of the atmosphere.

This paper gives an overview about DLR's current studies and developments with the goal to combine the advantages of FSO and RF-Communication. It discusses possible implementation concepts on different platforms and presents experimental results of the implemented FSO/RF hybrid communication system operating for airborne, optical downlinks at $1 \mathrm{Gbps}$.
\end{abstract}

Keywords: Free-Space-Optics, Laser-Communication, Hybrid-Link, high data-rate

\section{INTRODUCTION}

Optical communication links are an attractive solution in many application scenarios. The high data rate and power efficiency offered by optical communication systems enables overcoming bottlenecks introduced by classical (RF) communication systems. Data rates of $1 \mathrm{Gbit} / \mathrm{s}$ to $100 \mathrm{Gbit} / \mathrm{s}$ have been demonstrated in aircraft-to-ground and aircraftto-aircraft scenarios[1-3]. Optical data links were even investigated for building ad-hoc networks e.g. between airliners [4] and High Altitude Platforms (HAPs) [5], [6].

However, despite the high potential of the technology, optical data links suffer from limited availability due to atmospheric conditions and clouds. Especially in applications in which real-time data transmission of, for instance, aerial imagery for the monitoring of natural disasters, is required, optical data links cannot be applied alone. Therefore, Hybrid FSO/RF communication systems are of high interest. These combine the high availability of RF links with the high data rates of optical communications.

This paper will describe a Hybrid FSO/RF communication system which was developed within the DLR project $V A B E N E$, which aimed at monitoring and traffic management for mass events and natural disasters. An optical communication system, developed by the German Aerospace Center's Institute of Communications and Navigation (IKN), is combined with a high-resolution camera sensor, developed by the Institute of Remote Sensing (IMF). The design of the optical terminal and Hybrid FSO/RF link are described. Furthermore, results from a experimental campaign will be given.

*Benjamin.roediger@ dlr.de; phone +49 8153 28-2944; fax +49 8153 28-2844; dlr.de/kn/en/

Laser Communication and Propagation through the Atmosphere and Oceans IX, edited by Jaime A. Anguita,

Jeremy P. Bos, David T. Wayne, Proc. of SPIE Vol. 11506, 1150603 · C 2020 SPIE

CCC code: $0277-786 \mathrm{X} / 20 / \$ 21 \cdot$ doi: $10.1117 / 12.2567034$ 


\section{OPTICAL COMMUNICATION TERMINAL FELT II}

The Free-Space Experimental Laser Terminal (FELT II) was developed at DLR to research optical communication links in aeronautical scenarios. After the first flight of the system in 2008[7] it has been continuously further developed and was used in a number of application scenarios. This includes, for instance, projects researching traffic and disaster management [8], or the demonstration of aircraft-to-ground quantum key distribution[9].

For scenarios like the ones mentioned above a real-time communication is necessary. Modern sensor systems which can distinguish between relatively small objects like cars or people, even from a high altitude lead to high resolution pictures. This huge amount of data has to be transmitted to the ground in a shortness of time, for the security forces to react as soon as possible. Radio Frequency (RF) transmission is limited by bandwidth and represents the bottle neck in the communication from air to ground.

Free Space Optical (FSO) communication can provide higher data rates than RF communication and has the availability to break this bottleneck in bandwidth. Therefore IKN built the Free Space Experimental Laser Terminal (FELT-II) which provides a data transmission of up to $10 \mathrm{Gbit} / \mathrm{s}$.

The link is established using a Pointing Acquisition and Tracking (PAT) system. The sequence of events for link acquisition and tracking is as follows: first a beacon is activated by the Optical Ground Station (OGS) to illuminate the remote laser terminal, the laser terminal searches and acquires the signal. Then, the optical system of the FELT-II orientates itself to the beacon and starts the transmission via laser. This guarantees that the transmission laser is received by the ground station with a very high accuracy.

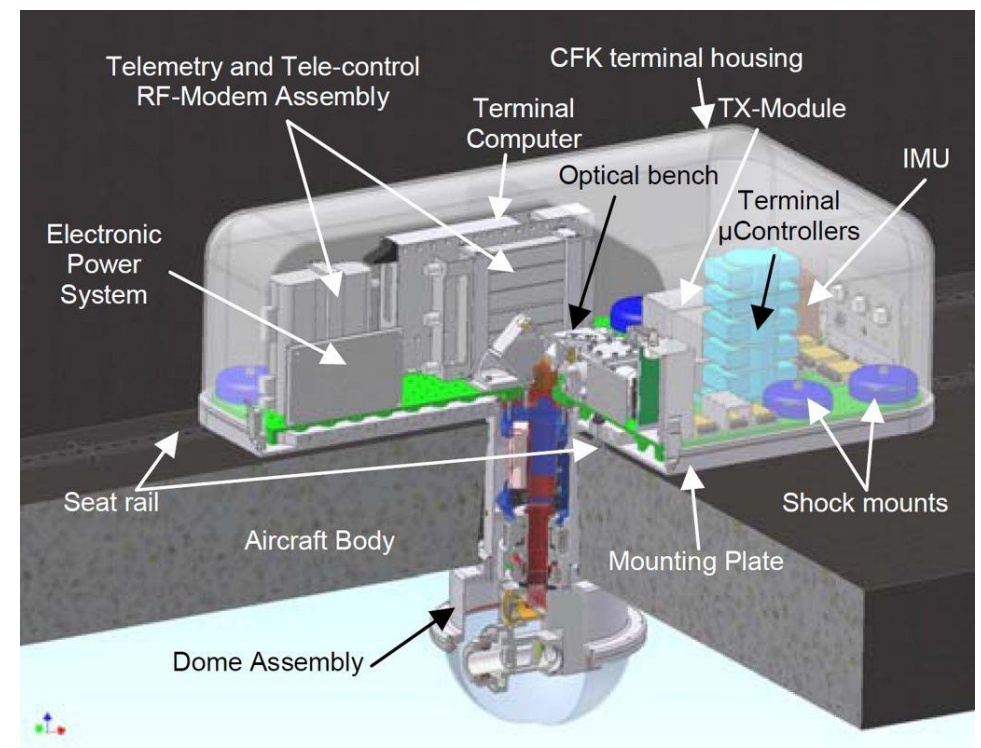

Figure 1. Cross cut of the FELT-II.

The cross cut of the 3D illustration of the FELT-II in Figure 1 shows the laser terminal with its main subsystems. The main part is the optical system. Inside the Dome Assembly a Coarse Pointing Assembly (CPA) is represented by a lens which is mounted with two motors movable in elevation and azimuth axis to be able to describe the whole hemisphere. Over a coudé-path the beam is lead to the inside of the terminal. Inside the Optical Bench a Fine Pointing Assembly (FPA) with a Fine Steering Mirror (FSM) improves the accuracy of the laser beam. As a sensor to measure the angular offset to the beacon a 4-Quadrant Diode (4QD) is used. To avoid differences between received beacon and transmission laser, the laser of the FELT-II is coupled into the same optical path.

\section{DATA-HANDLING}

The goal of IKN's contribution in the project is to provide a transparent and continuous communication between IMF data sources on the plane and its data sink on ground. Figure 2 shows the data transfer system between the IMF ground and air interfaces. 


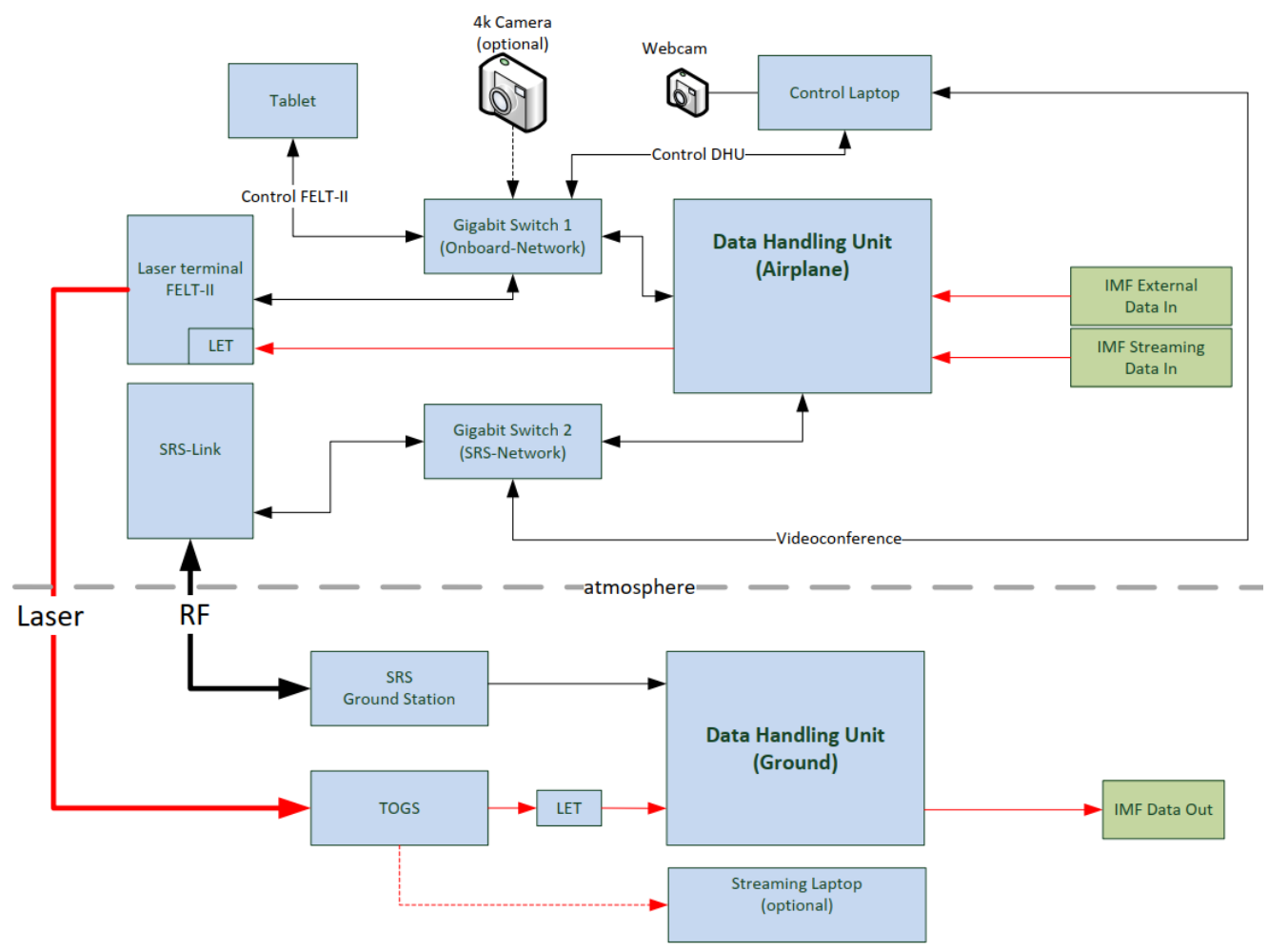

Figure 2. Block diagram of the data transfer system.

The block diagram shows the entire data transfer system with the transmission part on the air segment above and the receiving system on the ground segment below.

\subsection{FSO Communication}

Pictures taken by IMF's camera are processed onboard and sent to the Data Handling Unit (DHU). The DHU sends the data to a Laser Ethernet Transceiver (LET) which is proprietary DLR development [1]. The LET implements a FSO PHY layer including Forward Error Correction (FEC) and a communication protocol specially devised for data transmission through the atmosphere. Inside the FELT-II the electrical-optical conversion takes place and the data are sent via laser link to the ground. The laser is received by the Transportable Optical Ground Station (TOGS) where the optical-electrical conversion takes place. For the decoding another LET is used which forwards the raw data to the ground DHU and subsequently to the IMF data sink.

For the first tests and demonstrations of the entire transmission system, the IMF data source was replaced by 4k-camera. A live video out of the aircraft was streamed to the ground which proved the functionality of the whole transmission chain including the coding. After these first successful demonstrations, the quasi-operational data transfer including the IMF interfaces was demonstrated in several flight campaigns.

\subsection{RF Communication}

During the flight campaigns a direct communication between the teams in the aircraft and the ground station was necessary. Therefore DLR implemented a Radio Frequency (RF) communication system for this purpose. The Schulze Radio System (SRS) provides an RF-link with a data rate of up to $11 \mathrm{Mbit} / \mathrm{s}$ by using directional antennas on each side.

During the VABENE++ campaigns the SRS was exclusively used for communication via a video conference between the aircraft and the ground. After the successful demonstration of the laser link the idea arose to use this RF link to transmit the operational data in cases when the laser link is not available. 


\section{FSO RF HYBRID-LINK}

FSO provides a high license-free bandwidth throughput but it has more stringent requirements for pointing than classic $\mathrm{RF}$ communication. Additionally the atmosphere has a strong impact in the form of outages regarding the received optical power (in the order of milliseconds) that can lead to the loss of significant amount of data. On the other hand, classic RF communications provide a lower throughput but are more resilient to the pointing and atmospheric outages. In this scenario, the idea of combining both systems allows to take advantage of both techniques to ensure a minimum communication throughput while the optical channel is unavailable and a high throughput when FSO can resume normal operation. By adding bidirectional communications capabilities to the channel, the handover and switching between channels can be automated to further optimize the achieved throughput. The resulting Hybrid Link Protocol system comprises the implementation of a Hybrid ARQ (HARQ) with a rate less code and an algorithm for switching the link in correspondence to the measured state of the channel.

\subsection{Free Space Optical Channel}

The FSO links are affected by pointing errors due to limitations on the complex mechanisms required to established and maintain the optical links between the terminal and ground stations, as well as by atmospheric turbulence which manifests as scintillation in the received signal. Figure 3 present the measured BER at the receiver during a VABENE++ test flight, the observed data losses correspond to a combination of atmospheric effects, pointing errors and complete link disruptions lasting several seconds. The signal outages in the plot are identified by a BER value of 2, which is deliberately reported by the logging system in order to signal them indistinctly.

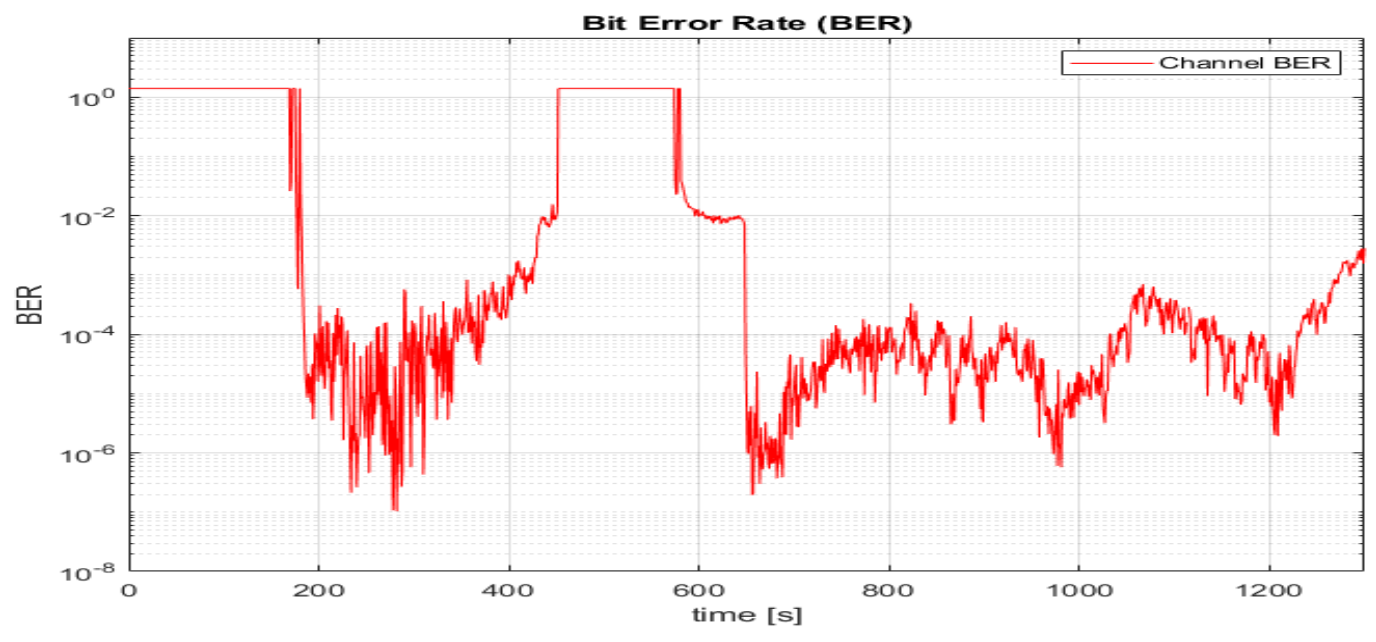

Figure 3. BER measured for and FSO downlink during a VABENE test flights. The BER over 1 indicate a Loss Of Signal.

\subsection{Communication Protocols}

Diverging properties and requirements on the RF and FSO links make necessary the implementation of different protocols for each channel. Therefore, Transmission Control Protocol (TCP) is used over the RF link. TCP offers additional reliability on the transport layer with its acknowledgement based communication. For the unidirectional FSO link, TCP is not directly applicable due to the missing feedback link, so that the unacknowledged User Data Protocol (UDP) is used on the FSO link to transport data. To ensure successful delivery of all transmitted packets over the FSO link, the RF feedback link is used to achieve a handshake as for Automated Repeat Request (ARQ) schemes. For each packet transmitted over the FSO channel, the receiver generates an acknowledgement message and transmits it back over the RF channel.

A major issue with this proposed handshake between two different links is the delay and corresponding round trip time (RTT). Since for this setup the FSO and RF links are implemented using different network interface cards (NIC), the feedback is only available after processing in the respective network stacks and operating system. Hence, using a simple stop-and-wait ARQ results in significant idle times. Consequently, it is proposed to apply a selective repeat scheme, where retransmission is only applied on erroneously received and lost packets with a sufficiently dimensioned sliding window size. However, since the FSO and RF links are not available at all time since they are prone to connection 
interruptions and loss of packets, a Hybrid ARQ (HARQ) [10] to protect data on the FSO link is introduced. HARQ applies concurrently ARQ and FEC to benefit from the high reliability from the former and the throughput in severe channel conditions from the latter.

In this specific application, the HARQ's design incorporates as FEC the RaptorQ code described in the IETF RFC 6330[11]. In general, HARQ can be split into two groups, Type I and Type II HARQ. In Type I, a fixed number of redundant symbols are added to each transmitted block. The receiver then tries to decode the block. If decoding is unsuccessful, a negative acknowledgement is created and the complete block is rejected. The rejected block including the redundant symbols is then requested by the receiver and accordingly retransmitted by the transmitter. This method is similar to the standard ARQ, where erroneous blocks are simply dropped as well. However, taking into consideration that the channel has a relatively long coherence time, the received data presents segments with a lot of burst errors along with error-free segments. Therefore, having a fixed number of redundant symbols during error free reception limits the throughput of the system according to the chosen code rate.

Higher throughput is achieved by using HARQ Type II, in which only the source symbols are transmitted initially. If all symbols arrive error-free at the decoder, original data can be recovered, otherwise a retransmission is requested and only then additional redundant symbols are transmitted to execute another decoding attempt. During stable signal conditions, Type II HARQ can hence perform with as high throughput as standard ARQ.

For this specific system, the following three conditions of operation are distinguished:

1. FSO and RF link are available: If both links are available, the system operates according to HARQ type II. Initially, the transmitter sends out source symbols over the FSO link. Each transmission is acknowledged over the RF feedback link. In case of a negative acknowledgement, the transmitter generates and sends out additional repair symbols until the data is decoded successfully.

2. Only FSO link available: Due to the missing RF feedback link, we cannot apply ARQ. The transmitter instead generates a fixed number of repair symbols and transmits them alongside the source symbols (as e.g. done in HARQ type I, but without any acknowledgement). We can configure the number of repair symbols arbitrarily, e.g. based on an estimation of the current channel condition.

3. Only RF link available: Since the RF link operates using TCP, which ensures acknowledged error-free reception at the receiver; no additional correcting measure on the transport layer is applied.

\subsection{System Coding}

In order to protect the data in the optical channel, the capabilities of the LET system are used. LET implements an $8 \mathrm{~b} / 10 \mathrm{~b}$ line coding in order to ensure a DC-balance in the transmission line and enough data transitions, required for the proper operation of the clock data recovery modules on the receiver side. The use of this coding also provides the advantage of identifying decoding errors which are then signaled as erasures to the FEC scheme thus improving its error correction capabilities.

This system implements a product code structure based on two Reed-Solomon (RS) codes. The outer code is a RS $(204,188)$ whereas the inner code is a RS $(204,180)$ and both use a symbol length of 8 bits. This scheme can be used stand-alone or in combination with an interleaver scheme that will offer protection against the outages in the optical channel. However, and for the sake of the protocol in this experiment the system was operating without interleaving in order to minimize the latency in the optical channel which can greatly affect the performance of TCP.

The physical layer code implemented on the LETs is capable of overcoming deep fades with duration of up to $20 \mathrm{~ms}$. However, during operation the system must also account for signal outages in which signal reception is not only impaired, but completely lost due to pointing errors. For use cases targeting delivery of large file sets, the aim is to ensure that all data is eventually received error-free. For this purpose, and as mentioned in section 4.2 additional errorcorrecting measures on the transport layer are implemented. Since the channel behavior is unpredictable and a suitable code-rate cannot be determined a-priori, we are introducing rate-less codes. Rate-less codes are a class of erasure codes that allows generating a potentially limitless sequence of encoded symbols from a set of source symbols. This code achieves constant per-symbol encoding/decoding cost with an overhead close to zero, especially when transporting large files. The advantage of the systematic property of the code is that encoded symbols are constituted by source symbols and redundant symbols. If we observe no error on the transport layer, i.e. all potential errors have been successfully recovered by the physical layer code on the LET, the source symbols are available at the receiver without decoding. If any error or frame loss occurred, the system can generate potentially limitless number of repair symbols to recover the original data in the decoding process. 


\subsection{Decision Algorithm}

The FSO and RF link of these set up are both potentially unreliable and might become unavailable during operation. In order to make use of all available transmission resources to maximize throughput, and ensure continuity in transmission, a link switching procedure between the two systems is proposed. The procedure applies a hard switching between the communications links, i.e. if the FSO is available, all data is transmitted over the optical link. In case the FSO link becomes unavailable, the system is able to redirect packets over the RF link to ensure an uninterrupted continuation of the transmission and to minimize any downtime. The design choice of hard switching over soft switching with continuous transmission over both links is motivated by the strong asymmetry in terms of throughput and jitter in arrival times of both links [5] [6]. These effects lead to increased decoder complexity and deliver only minimal throughput gain of soft switching compared to hard switching methods.

In order to detect availability of the links, we are utilizing heartbeat messages, i.e. packets with minimal payload transmitted in fixed cycles. If a certain number of these heartbeat messages are lost during transmission, the link is marked unavailable. The link status is continuously tracked over both links, based on which the link decision is made. It is important to mention that the selected link is subject to different communication protocols in correspondence with the three conditions of operation distinguished in section 4.2, namely: both links are available, only FSO link is available and only RF link is available.

\section{DEMONSTRATION MISSION}

\subsection{Test Setup}

The presented hybrid scheme was tested in the DLR campus in Oberpfaffenhofen, near Munich. The link was established at a static distance over 300 meters between the rooftop of the Institute of Communications and Navigation and a service tower in order to emulate a possible scenario with flying terminal (tower) and a ground station (rooftop) as Figure 4 presents. The transportable optical ground station (TOGS) [12] was placed on top of the building together with one of the RF antennas. The FELT-II terminal was placed by the tower alongside with the other antenna.

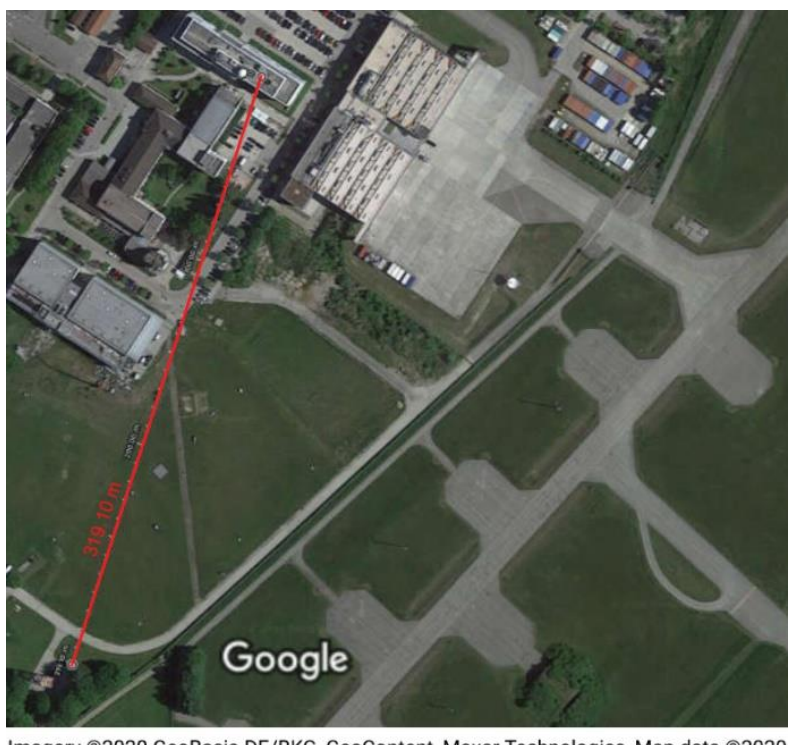

Figure 4 Demo location

Each terminal was accompanied with a data handling unit (DHU) a LET system and the support equipment to generate, transmit and visualize data as well as a control computer to monitor and control all the subsystems. Additionally, on the flying terminal, a fading testbed (FTB) [13] was included in order to achieve a more challenging channel and test the HARQ schemes. Figure 5 presents the block diagram of the main modules used for establishing the communication as well as the main communication channels used to interconnect them. 


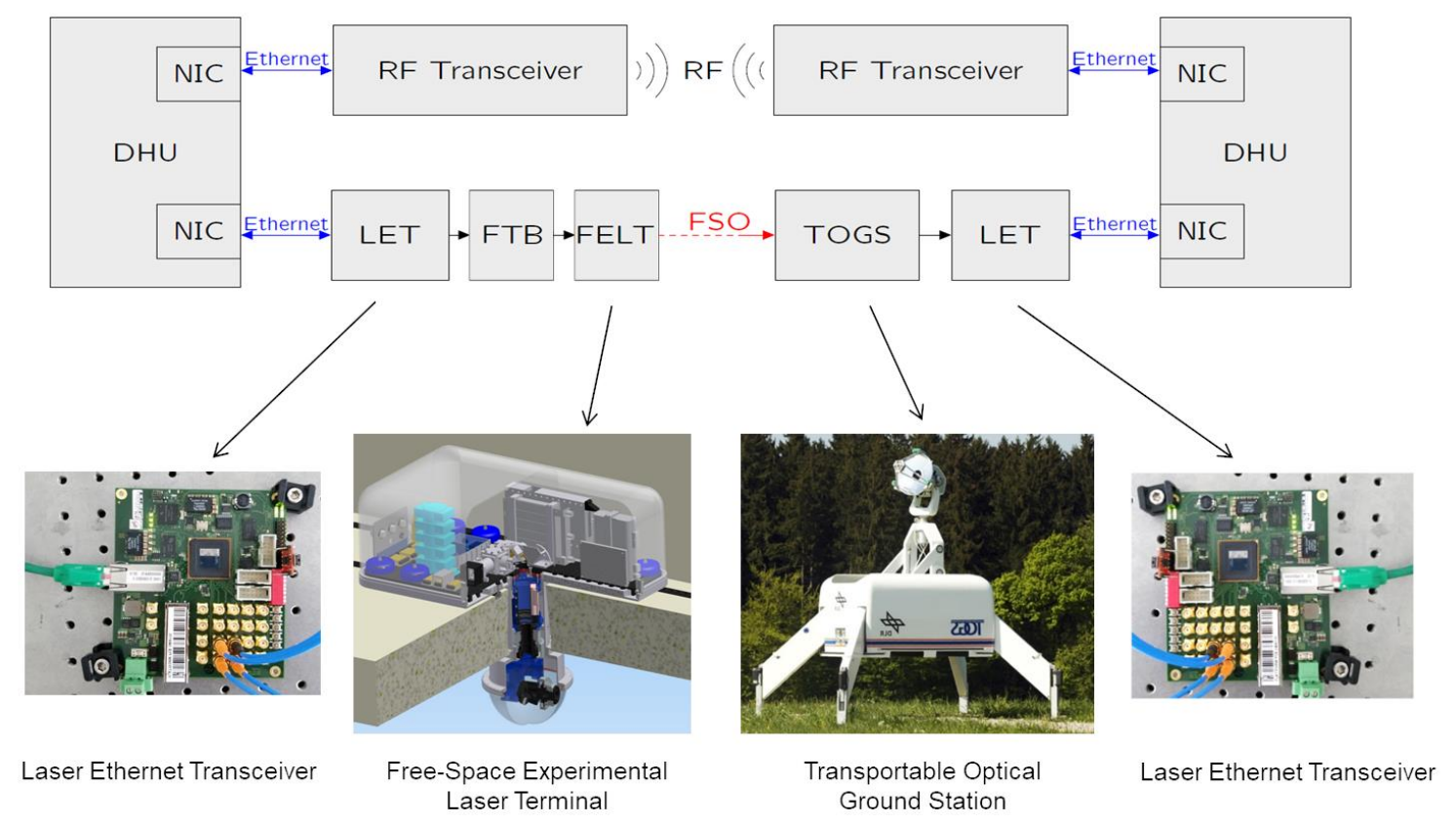

Figure 5 Demo setup equipment

In this configuration, a nominal throughput (at user level) was calculated for performance analysis. The LET system, through the FSO channel, offers a throughput of $610 \mathrm{Mbps}$. This throughput is partly limited by the Ethernet interface at the DHU side since both LET and DHU were characterized stand-alone and reported higher throughputs. Nonetheless, the value of $610 \mathrm{Mbps}$ is used for benchmarking the presented hybrid scheme. On the other hand, the RF antenna provides a nominal throughput of $5.8 \mathrm{Mbps}$ when used in the setup. Further analysis of its performance was considered non-relevant since the RF system is a $3^{\text {rd }}$ party component and there was no access to its internal design.

\subsection{Test Procedure}

We demonstrate a proof of concept by introducing deterministic fades to the optical channel with the help of a fading testbed. In this setup, we interrupt the optical link by introducing a strong attenuation emulating a loss of line of sight (LOS) communication, which results in burst errors of constant size. These attenuations were introduced using the FTB with a periodicity of 1 second and ranging from few milliseconds up to $200 \mathrm{~ms}$. In spite of the fact that the generated outages are unrealistic, these time durations were selected in order to test the system and validate the proposed scheme under very stringent conditions.

Under these conditions the system was tested with different HARQ and rate codes. The effectiveness of these different methods was measured at the DHU where the data logged from both terminals was post processed in order to determine how much data effectively transferred.

Furthermore the effectiveness of the switching procedure was assessed by introducing controlled outages to force the switching between the RF and FSO links. The transmitted and received data was once again logged at the DHU units and post processed in order to generate statistics for the analysis. 


\subsection{Results}

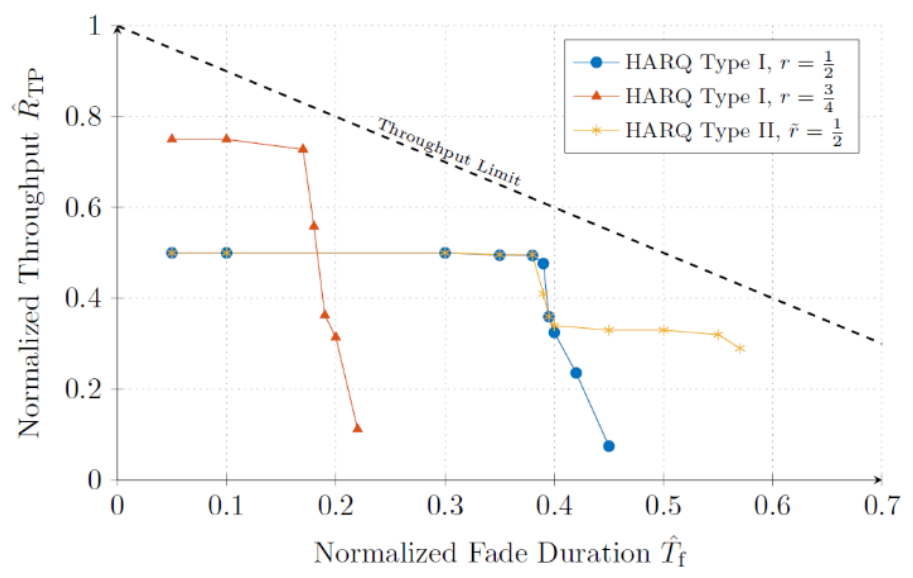

Figure 6 Normalized throughput of the HARQ with different code rates and high frequency fades

Figure 6 shows the achieved throughput for different HARQ and code rates under deterministic fades that are repeating with a cycle time $T_{c}=20 \mathrm{~ms}$. We configured the raptor code to transmit per code block $(\mathrm{k}=1000)$ a fixed number of redundant symbols, exemplarily with an effective code rate of $r=\frac{3}{4}$ and $r=\frac{1}{2}$ for the HARQ type I model. In this case, each transmission contains already all source and redundant symbols. If transmission fails, the whole block is rejected and a retransmission is initiated. In the HARQ type II case, only source symbols are transmitted initially. If decoding is unsuccessful, the same number of redundant symbols is transmitted, resulting in an effective rate of $\hat{r}=\frac{1}{2}$ after the first retransmission. The transmitter generates new redundant symbols of same block size until the receiver can decode the block error-free. The results show the performance of the used raptor codes in comparison to the theoretical limit of erasure codes. Given the rate-less property, we can design arbitrary code rates to achieve optimal performance under various link conditions.

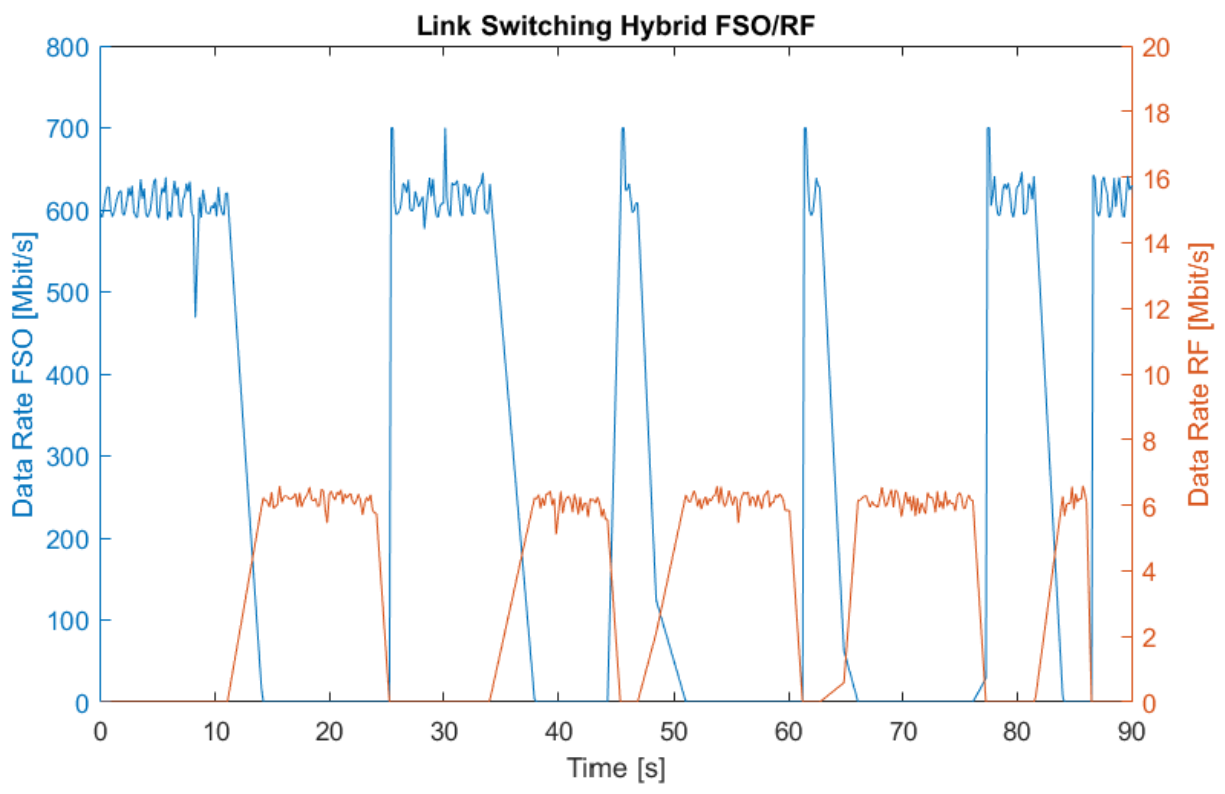

Figure 7 Switching performance of hybrid link 
Figure 7 shows the switching performance of our proposed system upon detection of a link outage. The interruption time depends on the chosen link availability detection frequency and timeout and can be tuned freely. In our test setup, we selected and configured a switching from FSO to RF after $2000 \mathrm{~ms}$ and vice versa after $350 \mathrm{~ms}$ upon link failure detection.

Finally, Table 1 presents the results of using raptor codes over the hybrid link. In this scenario, the switching between FSO and RF channels was disabled and the FSO channel was used for data transmission whereas the RF channel was used for control data. The data was encoded prior transmission, stored into a static memory and finally read out for transmission. On the receiver side, the decoding was performed in real time. A bug in this scheme is the responsible of the final rejected blocks presented by the table. The bug caused that the blocks marked for retransmission were not properly read from the memory and therefore they were not being retransmitted. After the campaign, the source of this issue was identified, corrected and its solution verified in the laboratory.

Table 1 Summary of the Fountain codes results

\begin{tabular}{|c|c|c|}
\hline & Short block & Long block \\
\hline Sent Bytes & 2.101 .984 .280 & 2.103 .461 .568 \\
\hline Received Bytes & 2.09 .725 .596 & 2.102 .519 .484 \\
\hline Lost Bytes & 3.258 .684 & 942.084 \\
\hline Block Size & 250 symbols & 1000 symbols \\
\hline Symbol Size & 1400 bytes & 1400 bytes \\
\hline Total Blocks & 4233 & 1101 \\
\hline Total Transmissions & 4237 & 1101 \\
\hline Received Blocks & 4233 & 1101 \\
\hline Acknowledged Blocks & 4228 & 1098 \\
\hline Rejected Blocks & 5 & 3 \\
\hline Decoding Error & $1.1 \times 10^{-3}$ & $2.7 \times 10^{-3}$ \\
\hline Code Rate & 0.5 & 0.5 \\
\hline Total Files & 111 & 111 \\
\hline $\begin{array}{l}\text { Successfully Decoded } \\
\text { Files }\end{array}$ & 107 & 108 \\
\hline
\end{tabular}

\section{APPLICATION TRANSFER TO OTHER PLATFORMS}

FSO provides a lot of advantages for multiple communication platforms. The demands of high data rates and secure communication channels rise in all communication fields. Nevertheless, robust links and a high availability are still requested and often assumed as given. FSO is a useful and sometimes mandatory extension to RF-communication. Despite the advantages of laser-communication it is dependable on high precision mechanisms and a direct line of sight. If these preconditions cannot be guaranteed switching to a more robust link like RF is necessary, if lower data throughputs can be accepted. In most applications FSO cannot replace RF-communication completely, which leads to Hybrid-links as a logical consequence. 


\subsection{Necessity of Hybrid Links in other platforms}

The implementation of Hybrid-links depends on the carrier platform and the usage. FSO can be used wherever high amounts of data have to be transmitted. This can be scientific measurement data or multimedia data like pictures or videos. On airplanes, drones or satellites sensor data have to be transmitted in real-time. These data are uncritical for the operation of the platform itself. Whenever low data rates are sufficient but a high availability is mandatory the RFchannel will still be used. This can be telemetry and telecommand (TM/TC) for example.

When data shall be distributed over large distances like High Altitude Platforms (HAP's) or satellite backbones, FSO and $\mathrm{RF}$ channels can be used for transmitting the same data. In this scenario FSO is the main channel and RF is the fall back technology if FSO is not available. The data can still be transmitted but compromises have to be made. These can be a lower data rate which leads into time delays, higher compression of the data or reduced quality.

Furthermore, due to the low divergence, robustness against jamming, spoofing and eavesdropping FSO can also be used for high sensible data. Especially in military or financial applications the high secure FSO channel shows its advantages towards RF-communication. Critical or secret data can be transmitted via FSO over a high secure channel. In combination with Quantum Key Distribution (QKD) the communication reaches its maximum secure level.

\subsection{Outlook}

The advantages of optical-RF-Hybrid-links have been presented in this paper. Also optical downlinks from satellite platforms suffer from a limited availability of optical links due to atmospheric effects. Especially in scenarios with a high required availability, hybrid links can help to overcome the limiting factors of the mission. For remote sensing and earth observation, the time to access the images is essential, especially in emergency missions like fire monitoring or after natural catastrophes. A hybrid link on a small spacecraft can support a high data rate during periods of stable optical link availability while the RF-link is insensitive against atmospheric effects and can bridge the gap in communication.

\section{REFERENCES}

[1] J. Horwath, D. D. Gonzalez, L. M. Navajas, A. L. Souto, V. Semerjyan, G. Raju, J. Grabowsky, C. Garcia, Y. Lai, T. Bähr, and others, "Test results of error-free bidirectional $10 \mathrm{Gbps}$ link for air-to-ground optical communications," in Free-Space Laser Communication and Atmospheric Propagation XXX, 2018, vol. 10524, p. 105241F.

[2] F. Moll, J. Horwath, W. Mitzkus, A. Shrestha, M. Brechtelsbauer, C. Fuchs, M. N. Luis, L. S. Alberto, and D. G. Dionisio, "Demonstration of high-rate laser communications from a fast airborne platform," IEEE Journal on Selected Areas in Communications, 2015.

[3] C. Chen, A. Grier, M. Malfa, E. Booen, H. Harding, C. Xia, M. Hunwardsen, J. Demers, K. Kudinov, G. Mak, and others, "Demonstration of a bidirectional coherent air-to-ground optical link," in Free-Space Laser Communication and Atmospheric Propagation XXX, 2018, vol. 10524, p. 105240G.

[4] K.-D. Büchter and A. Sizmann, "Feasibility of high-speed transparent photonic links in airborne free-space optical communication," in Avionics, Fiber-Optics and Photonics Conference (AVFOP), 2013 IEEE, 2013, pp. 19-20.

[5] J. Horwath, M. Knapek, B. Epple, M. Brechtelsbauer, and B. Wilkerson, "Broadband backhaul communication for stratospheric platforms: the stratospheric optical payload experiment (STROPEX)," in SPIE Optics+ Photonics, 2006, p. 63041N-63041N.

[6] B. Moision, B. Erkmen, E. Keyes, T. Belt, O. Bowen, D. Brinkley, P. Csonka, M. Eglington, A. Kazmierski, N. Kim, and others, "Demonstration of free-space optical communication for long-range data links between balloons on Project Loon," in Free-Space Laser Communication and Atmospheric Propagation XXIX, 2017, vol. 10096, p. $100960 Z$.

[7] J. Horwath and C. Fuchs, "Aircraft to Ground Unidirectional Laser-Comm. Terminal for High Resolution Sensors," in Free-Space Laser Communication Technologies XXI, Vol 7199, 2009, no. 7199-1.

[8] C. Fuchs, C. Schmidt, B. Rödiger, A. Shrestha, M. Brechtelsbauer, J. R. Molina, J. Pacheco, and V. Gstaiger, "DLR's Free Space Experimental Laser Terminal for Optical Aircraft Downlinks," in Proc. of SPIE Vol, 2017, vol. 10096, pp. 1009610-1.

[9] S. Nauerth, F. Moll, M. Rau, C. Fuchs, J. Horwath, S. Frick, and H. Weinfurter, "Air-to-ground quantum communication," Nature Photonics, 2013. 
[10] S. B. Wicker, Error control systems for digital communication and storage, vol. 1. Prentice hall Englewood Cliffs, 1995.

[11] M. Luby, A. Shokrollahi, M. Watson, T. Stockhammer, and L. Minder, "RaptorQ forward error correction scheme for object delivery-rfc 6330," IETF Request For Comments, 2011.

[12] C. Fuchs, M. Brechtelsbauer, J. Horwath, A. Shrestha, F. Moll, D. Giggenbach, and C. Schmidt, "DLR's Transportable Optical Ground Station," in OSA Technical Digest (online), 2013.

[13] A. Shrestha, D. Giggenbach, A. Mustafa, J. Pacheco-Labrador, J. Ramirez, and F. Rein, "Fading testbed for freespace optical communications," in Advanced Free-Space Optical Communication Techniques and Applications II, 2016, vol. 9991, p. 999105. 